\title{
In Vitro Low Responsiveness of Leishmania donovani Towards Sodium Antimony Gluconate
}

\author{
Murshed Alam¹, AKM Shamsuzzaman², AKM Musa², Abul Hossain Khan¹, Md. Chand Mahmud², Mesbah Uddin Ahmed², Md. \\ Akram Hossain², Abdullah Akhtar Ahmed ${ }^{2}$
'Department of Microbiology, Community Based Medical College, Mymensingh; 2Department of Microbiology, Mymensingh Medical College, Mymensingh; ${ }^{3}$ Department of Microbiology, Kumudini Medical College, Mirzapur, Tangail

\begin{abstract}
Kala-azar has been uprising concomitantly with drug-resistant strains of the causatinve agent, particularly in the neighbouring India. The actual perspective of drug resistance in Leishmania donovani in Bangladesh is yet to be explored. So, this prospective study, as a preliminary one, was done to observe in vitro drug responsiveness against Sodium Antimony Gluconate (SAG) and Amphotericin B of 41 strains of L. donovani isolated from Kala-azar cases. The cases $(n=41)$ were selected from 45 clinically suspected febrile patients those who were positive for Kala-azar by immunochromatographic test (ICT). The selected cases were subsequently confirmed as Kala-azar by detection of Leishmania Donovan (LD) bodies from bone marrow aspirates $(\mathrm{n}=38)$ by microscopy and/or showing promastigotes in modified McNeal, Nicole and Novy (NNN) media ( $n=41)$. Minimum Inhibitory Concentrations (MICs) of SAG and Amphotericin B were seen in relation with history of previous SAG therapy of the patients. Among 08 strains with previous SAG therapy, MICs of SAG were $500 \mu \mathrm{g}$ in $05(62.5 \%)$ and $250 \mu \mathrm{g}$ in $03(37.5 \%)$ cases. In remaining 33 strains with no previous SAG therapy, MIC of the drug was $250 \mu \mathrm{g}$. In all 41 strains, MIC of Amphotericin B was 05 $\mu \mathrm{g}$ irrespective of the history of previous SAG therapy. The study revealed that strains of $L$. donovani with low responsiveness to standard dose of pentavalent antimonials have been started to appear in our community that needs further study at community level in a larger population.
\end{abstract}

Key words: Leishmania donovani, Low responsiveness, Sodium antimony gluconate

\section{Introduction}

Sodium antimony gluconate (SAG) was first introduced in the decade of 1940 and used as a first-line drug for the treatment of Visceral Leishmaniasis (VL). The drug is administered parenterally at a dose of $20 \mathrm{mg} / \mathrm{kg}$ body weight daily for 20-40 days. ${ }^{1}$ If treatment with SAG is unsuccessful, Pentamidine is used, administered parenterally at a dose of 2$4 \mathrm{mg} / \mathrm{kg}$ body weight 1-3 times a week. ${ }^{2}$ Amphotericin B is another drug of second choice administered as a slow

\footnotetext{
Correspondence:

Dr. Murshed Alam

Assistant Professor, Department of Microbiology,

Community Based Medical College, Mymensingh
}

intravenous infusion of $2 \mathrm{mg} / \mathrm{kg}$ body weight on alternate days for 20-40 doses. Lipid associated drug (Ambisome) is the Amphotericin B incorporated into Liposomes and has been used successfully to treat Kala-azar patients unresponsive to standard drugs. ${ }^{3}$ The Ambisome is administered intravenously at $3 \mathrm{mg} / \mathrm{kg}$ body weight daily for 30 days in a $5 \%$ glucose solution as a slow drip. Ambisome can be used for all ages. ${ }^{4}$ Recently, Miltefosine appeared to be the first orally effective agent for the treatment of VL given at a dose of $100 \mathrm{mg} /$ day for 28 days for adults weighing more than $25 \mathrm{~kg} .{ }^{5}$ The drug has already been registered in India since March, 2001. Other drugs under investigation are Paromomycin, Imidazoles, Triazoles and Purine analogueAllopurinol. Paromomycin, an antibiotic of the 
Aminoglycoside family was used in India and Kenya in combination with Sodium Stibogluconate $(\mathrm{Sb})$ and found effective in treating unresponsive cases of VL.6,7

Major limitations considered in the treatment of Kala-azar are various side effects of the drugs and treatment failure. Up to $15 \%$ therapeutic failure occurs with antimonials worldwide. But, one study in Bihar, India documented treatment failure rate of $65 \%$ and two other studies recorded treatment failures at a rate of $37 \%$ and $42 \% .^{8-10}$ Explanations of Antimony treatment failures include under-treatment due to inadequate supply of the drug and immunologic or pharmacokinetic defects in the host. ${ }^{11}$ In Bangladesh, rate of treatment failure is roughly estimated as $5 \%, 12$ which does not simulate with the clinical experiences and is expected to be higher. The hypothesized event of treatment failure is supposed to be due to emergence of resistance properties among strains of $L$. donovani against SAG. This would be a serious threat for public health in near future since incidence of Kala-azar has been uprising concomitantly with drug resistant strains of $L$. donovani. ${ }^{11,12}$ So, it is an essential demand of time to elucidate the actual perspectives of drug-resistance in L. donovani. In this context, no study yet has been done or reported from Bangladesh.

Considering the background described above, any preliminary study on drug responsiveness of VL cases would stand rational for overall public health ground. And further advanced studies would be possible taking information from a base-line study. Therefore, the present study was designed to find out drug responsiveness among L. donovani of VL cases.

\section{Methods}

This prospective study was conducted in the Department of Microbiology, Mymensingh Medical College during the period from March, 2004 to February, 2005. Cases were selected from Mymensingh Medical College Hospital, Community Based Medical College Hospital and nearby Trishal Thana Health Complex. Relevant history, clinical findings, laboratory records and findings of follow-up of every case was recorded in a pre-designed data sheet and subsequently analyzed by computer programme SPSS version 12.0 .

Clinically suspected febrile patients on the basis of prolonged low-grade fever, weight loss, hepato-splenomegaly, anemia with or without skin pigmentation, and history of previous SAG therapy (treatment failure) $(n=45)$ were investigated for anti-leishmanial antibodies using K-39 antigen-based Immunochromatographic test (ICT). Cases showing ICTpositive results were subsequently confirmed as Kala-azar by detection of LD bodies from bone marrow aspirates by microscopy and/or showing growth of promastigotes in culture $(n=41)$.

\section{Collection of bone marrow and culture into NNN media:}

$\mathrm{Up}$ to $1 \mathrm{ml}$ of bone marrow was collected from iliac crest of each of the study cases at Microbiology departments of the study sites following standard procedure. Immediately after aspiration of bone marrow, thin films of the aspirate were made on at least three microscopic slides. At the same time, one drop of the marrow materials was inoculated into the water of condensation of two sets of tubes of the NNN media: one set of drug free media and another containing specific drugs following standard procedure.13 After inoculation of marrow material into 2 drug free NNN tubes and 3 tubes of different strengths of each SAG $(0.25 \mathrm{mg} / \mathrm{ml}$, $0.5 \mathrm{mg} / \mathrm{ml}$ and $1.0 \mathrm{mg} / \mathrm{ml})$ and Amphotericin B (05 $\mu \mathrm{g}, 10$ $\mu \mathrm{g}$, and $15 \mu \mathrm{g} / \mathrm{ml}$ ) were incubated at $24^{\circ} \mathrm{C}$ in a thermostatregulated refrigerator in the range of $18-25^{\circ} \mathrm{C} .{ }^{14}$

\section{Staining and microscopic examination of marrow film:}

The bone marrow films were stained with Leishman stain according to the previously described method. ${ }^{15}$ At least 1000 fields per slide, preferably around the edges of the preparation, were examined to detect amastigote of Leishmania donovani. If any amastigote form was found in 1000 fields, the slide was reported as positive. Grading of the positive smears was done according to previously developed standard chart. ${ }^{16}$

Observation for Promastigotes: Promastigotes of $L$. donovani were detected from water of condensation of each of properly inoculated and incubated culture media by observing typical morphology and motility. On finding promastigotes in any such preparation, the corresponding 
culture result was reported as positive. This procedure was started from the 3rd day of incubation onwards up to 7 days. Tubes showing no promastigotes within 7 days, kept incubated further upto 10 days. On 10th day, if no promastigote was found again, the tubes were reported as negative and discarded. ${ }^{16}$

Reporting of drug sensitivity: If any promastigote was seen in any drug-containing tube, the parasite was designated as unresponsive to that particular concentration of the drug. ${ }^{14}$

\section{Results}

The present study was conducted on a total of 45 subjects. Of them, 41 (91.11\%) were confirmed as Kala-azar cases having parasite-positive laboratory findings. Majority of the cases $(29,64.4 \%)$ were in the age group of 2-12 years. Number of cases were found to decline gradually with increase in age. A minimum of $01(2.44 \%)$ case was in the age group of 57 years and above. Total males were 27 (60.0\%) and females $18(40.0 \%)$ (Table I) giving a male to female ratio of $1.5: 1$.

The relationship between history of previous SAG therapy and in vitro $\mathrm{MIC}$ of the isolates of $L$. donovani promastigotes was considered and it was seen that MIC was higher among previous SAG history-positive cases. Out of 08 historypositive cases, the MIC of SAG was $500 \mu \mathrm{g} / \mathrm{ml}$ in 05 cases and $250 \mu \mathrm{g} / \mathrm{ml}$ in 03 cases, whereas in the remaining 33 history-negative cases, the MIC of SAG was $250 \mu \mathrm{g} / \mathrm{ml}$. (Table II)

Table I: Age and gender distribution of study subjects

\begin{tabular}{lcc}
\hline Age in years & \multicolumn{2}{c}{ Gender of the cases } \\
& Male & Female \\
\hline $2-12(\mathrm{n}=29)$ & 17 & 12 \\
$13-23(\mathrm{n}=07)$ & 04 & 03 \\
$24-34(\mathrm{n}=03)$ & 01 & 02 \\
$35-45(\mathrm{n}=03)$ & 02 & 01 \\
$46-56(\mathrm{n}=02)$ & 02 & 00 \\
$>56(\mathrm{n}=01)$ & 01 & 00 \\
Total $(\mathrm{n}=45)$ & 27 & 18 \\
\hline
\end{tabular}

Table II: Relationship between treatment failure and MIC of SAG

\begin{tabular}{llll}
\hline Treatment failure & \multicolumn{3}{c}{ MIC of the $L$. donovani promastigotes } \\
& $250 \mu \mathrm{g} / \mathrm{ml}$ & $500 \mu \mathrm{g} / \mathrm{ml}$ & $1000 \mu \mathrm{g} / \mathrm{ml}$ \\
\hline Yes $(\mathrm{n}=08)$ & $03(37.5 \%)$ & $05(62.5 \%)$ & $00(00 \%)$ \\
No $(\mathrm{n}=33)$ & $33(100.0 \%)$ & $00(00 \%)$ & $00(00 \%)$ \\
Total $(\mathrm{n}=41)$ & $36(87.8 \%)$ & $05(12.2 \%)$ & $00(00 \%)$ \\
\hline
\end{tabular}

No history of previous Amphotericin B therapy was recorded. In all 41 cases, the MIC of Amphotericin B was $05 \mu \mathrm{g} / \mathrm{ml}$.

\section{Discussion}

Chemotherapy is critically important in reducing the burden of disease, and antimonials (SbV) are the first-line drugs for all clinical forms. Treatment by $\mathrm{SbV}$ is long, expensive and not devoid of adverse side effects. In many states of India, treatment failure is already well documented due to low responsiveness of parasites to sodium antimony gluconate (SAG). In Bangladesh, resurgence of Visceral Leishmaniasis (VL) has been noticed first in the decade of 1970 that has gradually increased to an epidemic form in many localities. Currently, VL appears at a rate in excess of 15,000 cases per year. ${ }^{12,17}$

Kala-azar occurs among various age groups depending on the infecting species, geographic location, disease-reservoir and host immunocompetence. In Indian type of VL, children between 5 and 15 years of age were affected more. ${ }^{17}$ In the present study, majority of cases (64.4\%) were in the 2-12 years age group. Almost similar findings were also found in many studies. Ali and Ashfold in a study found 142 cases of VL, where $58 \%$ were children below 15 years. ${ }^{18}$ In another study by Mittal et al observed that majority patients of VL were in the age group of 5-15 years. ${ }^{19}$ Higher incidence of KA in children might be due to the observation that children of poor family generally suffer from malnutrition. Consequently, their immunity is hampered, increasing the risk of getting KA infection and developing severe disease. Moreover, adult population in the endemic locality might develop protective immunity from previous infection that reduces the chance of re-infection. ${ }^{20}$ 
In the present study, male to female ratio was $1.5: 1$. Other two reports in home from the ICDDR,B also reported almost similar ratio between male to female and found the ratio of 1.2:1 and 1.04:1.21,22 Several local studies were also consistent as showing male to female ratio of $1.38: 1,2: 1$, and $2.4: 1.17,23$ Similar finding was reported also from abroad where ratio between male to female was 1.3: 1.24 In a study from Sudan, it was reported that like many other countries males are almost twice $(1.8: 1)$ as likely to be affected by VL than females. ${ }^{25}$ Males are infected more often than females, most likely because of their increased exposure to sand flies due to professional activities.

In the present study, 41 strains of Leishmanial parasites were isolated in culture. Out of which 36 strains did not yield promastigotes at a SAG concentration of $250 \mu \mathrm{g} / \mathrm{ml}$ (MIC). Of 8 strains isolated from treatment failure KA cases with previous SAG therapy, 05 did not yield promastigotes at SAG concentration of $500 \mu \mathrm{g} / \mathrm{ml}$ (MIC).

As a whole, about $12.2 \%(05 / 41)$ strains showed higher inhibitory concentration of SAG in the present study. On the contrary, none of 41 strains yielded promastigotes at an MIC of Amphotericin B of $5 \mu \mathrm{g} / \mathrm{ml}$. We could not compare this data with that of other studies at home. Because, no data in this respect is as yet published or reported. But studies from India indicated that unresponsiveness to pentavalent antimonials had increased from $34 \%$ to $64 \%$ recently. ${ }^{10,14}$ Parasites causing Kala-azar among those who were clinically unresponsive also showed resistance in in vitro sensitivity test method using standard concentration of pentavalent antimonials. Besides these, it was further observed that pentavalent sensitive parasites could be made resistant to the drug by repeated passage in experimental animals followed by incomplete treatment with suboptimal doses of the drug. ${ }^{12}$ Considering above-mentioned observations, it was suggested that the steady rise in pentavalent antimony unresponsiveness of KA patients as found in India were due to infection with resistant parasites, generated as a result of irregular and often incomplete treatment of the patients. Since the present study could not investigate all those parameters, no comment could be made. But irregular and incomplete treatment by SAG in the poor KA patients in Bangladesh is a common phenomenon due to nonavailability and high cost of the drug. ${ }^{12}$ Considering the statement, higher MIC for Leishmanial isolates from treatment failure KA cases obtained in the present study might be logical. But the issue needs critical and urgent exploration on a large- scale study to encounter prevailing and future surgence of KA all over the country.

Analyzing the findings of the study, it can be concluded that KA affected more males than females and majority of the infections occurring among children were under 12 years of age. Strains of $L$. donovani unresponsiveness to standard dose of pentavalent antimonials have been started to appear in Bangladesh.

\section{References}

1. Hashim FA, Ali MS, Satti M, et al. An outbreak of acute Kala azar in a nomadic tribe in western Sudan: features of the disease in a previously non-immune population. Trans R Soc Trop Med Hyg 1995; 88: 431-432.

2. WHO expert committee report. Control of the Leishmaniasis Technical Report Series no. 793. Geneva: WHO; 1991: pp. 119.

3. Olliaro PC, Bryceson ADM. Practical progress and new drugs for changing patterns of Leishmaniasis. Parasitol Today 1993; 9: 323-328.

4. Dietze R, Fagundes SM, Brito EF, et al. Treatment of Kala-azar in Brazil with Amphocil (amphotericin B cholesterol dispersion) for 5 days. Trans R Soc Trop Med Hyg 1995; 89: 309-311.

5. Sundar S, Jha TK, Thakur CP, Engel J, Sindermann H, Fischer C. Oral miltefosine for Indian leishmaniasis. N Engl J Med 2002; 347: 1739-1746.

6. Thakur CP, Bhowmick S, Dolfi L, Olliaro P. Aminosidine plus sodium stibogluconate for the treatment of Indian kala azar: a randomized dose finding clinical trial. Trans R Soc Trop Med Hyg 1995; 89: 219-223.

7. Bryceson ADM. Leishmaniasis. In: Mansoon's tropical diseases, 20th edn. Philadelphia: WB Saunders Co. Ltd.; 1996: pp. 12131245.

8. Sundar S, Reed SG, Singh VP, Kumar PCK, Murray HW. Rapid accurate field diagnosis of Visceral Leishmaniasis. Lancet 1998; 351: 563-565.

9. Jha TK, Olliaro P, Thakur CP, et al. Randomised controlled trial of aminosidine (paromomycin) $\mathrm{v}$ sodium stibogluconate for treating Visceral Leishmaniasis in North Bihar, India. Br Med J 1998; 316: 1200-1205.

10. Thakur CP, Sinha GP, Pandey AK. Do the diminishing efficacy and increasing toxicity of sodium stibogluconate in the 
treatment of Visceral Leishmaniasis in Bihar, India; justify its continued use as a first-line drug? An observational study of 80 cases. Ann Trop Med Parasitol 1998; 92: 561-569.

11. Lira R, Sundar S, Makharia A, et al. Evidence that the high incidence of treatment failures in Indian Kala-azar is due to the emergence of antimony-resistant strains of Leishmania donovani. J Infect Dis 1999; 180: 564-567.

12. Directorate of Health Services. Kala-azar: A training module for medical officers. Dhaka: DGHS; 1995: pp. 1-30.

13. Carreina PF, Maingonr, Ward RD, et al. Molecular Techniques in the Characterization of Leishmania Isolates from Central America. Ann Trop Med Parasitol 1995; 89: 31-36.

14. Bhattacharyya A, Mukherjee M, Dattagupta S. Studies on Stibanate unresponsive isolates of Leishmania donovani. J Biosci 2002; 27: 503-508.

15. Duguid JP. Staining methods- Leishman's stain. In: Mackie \& McCartney- Practical Medical Microbiology, 14th edn. New York, USA: Macgraw-Hill Companies Inc.; 1996: pp. 808.

16. World Health Organization. Manual on Visceral Leishmaniasis Control, Geneva: Division of control of Tropical Diseases; 1996 (WHO/ Leish/ 96.40): pp. 1-84.

17. Talukder SI, Huq MH, Rahman S, Haque MA, Sarker CB, Ali MS. Epidemiological characteristics of sixty five cases of Kalaazar attending to a laboratory in Mymensingh. Mymensingh Med J 2003; 12: 89-92.

18. Ali A, Ashford RW. Visceral leishmaniasis in Ethiopia. III. The magnitude and annual incidence of infection, as measured by serology in an endemic area. Ann Trop Med Parasitol 1994; 88: 43-47.

19. Mittal V, Singh VK, Ichhpujani RL. Kala-azar in Uttar Pradesha study of 66 patients. J Commun Dis 2002; 34: 43-49.

20. Berman JD. Human Leishmaniasis: Clinical, diagnostic and chemotherapeutic development in the last 10 years. Clin Infect Dis 1997; 24: 684-703.

21. International Centre for Diarrhoeal Disease Research in Bangladesh (ICDDR,B). Visceral Leishmaniasis, Mymensingh, 2002. Health Sc Bull 2003; 1 (2): 1-6.

22. International Centre for Diarrhoeal Disease Research in Bangladesh (ICDDR,B). Clinical characteristics of Visceral Leishmaniasis in an Endemic Community in Bangladesh. Health Sc Bull 2002; 3: 6-9.

23. Sarker CB, Momen A, Jamal MF, Siddiqui NI, Siddiqui FM, Chowdhury KS. Immunochromatographic (rK39) strip test in the diagnosis of Visceral Leishmaniasis in Bangladesh. Mymensingh Med J 2003; 12: 93-97.

24. Gradoni L, Bryceson A, Desjeux P. Treatment of Mediterranean Visceral Leishmaniasis. Bull World Health Organ 1995; 73: 191-197.

25. Zijlstra EE, El-Hassan AM, Ismael A, Ghalib HW. Endemic Kala-azar in eastern Sudan: a longitudinal study on the incidence of clinical and subclinical infection and post-Kalaazar dermal leishmaniasis. Am J Trop Med Hyg 1994; 51: 826836. 